\title{
EVN AS A PHASE STABLE INTERFEROMETER
}

\author{
LARS B. BÅÅTH \\ Onsala Space Observatory, S-439 00 Onsala, Sweden \\ FRANCO MANTOVANI \\ Istituto di Radioastronomia, via Irnerio, Bologna, Italy
}

\begin{abstract}
A technique has been developed by which the data from the Mk3 correlator are read directly into the AIPS package. The data are Fourier transformed from delay lags into frequency channels and kept separately for each IF channel. The data are then corrected for multiand single band delays. We have used this technique to solve for fringe solutions on a reference source and then apply the solution to correct the data on the target source. In a recently analysed experiment we managed in this way to phase reference, using the European VLBI Network (EVN) at $18 \mathrm{~cm}$, the quasar OQ208 and the radio quasar $1422+202$. These sources are separated by more than $9^{\circ}$ on the sky.
\end{abstract}

\section{INTRODUCTION}

A phase stable interferometer has the advantage over a VLBI system that coordinates of a source on the sky can be obtained directly once the system has been phased up towards a set of calibration sources. In VLBI we have to use various clever schemes to connect the phases between two sources which are relatively close on the sky. The distance over which the phases can be connected is limited by the atmosphere: the ionosphere at low frequencies and the troposphere at higher frequencies. The limit is usually quoted to be about 30'. This limit is larger for more compact VLBI arrays, as the European VLBI Network (EVN), and our data suggest it can be extended to as much as $10^{\circ}$ at night time.

\section{PHASE REFERENCING THE PAIR OQ208/1422+202}

This experiment started as an attempt to apply the "Wide Field Mapping" technique (see Okopi and Båath these proceedings) on VLBI data. The source $1422+202$ had previously been observed with the EVN by Franco Mantovani. The data showed evidence of beating from two components separated by about 8 ". One of the components was very weak and the beating could only be detected on the short and very sensitive baseline Effelsberg - Westerbork. We decided to make a new attempt with the Mk3 system to increase the 


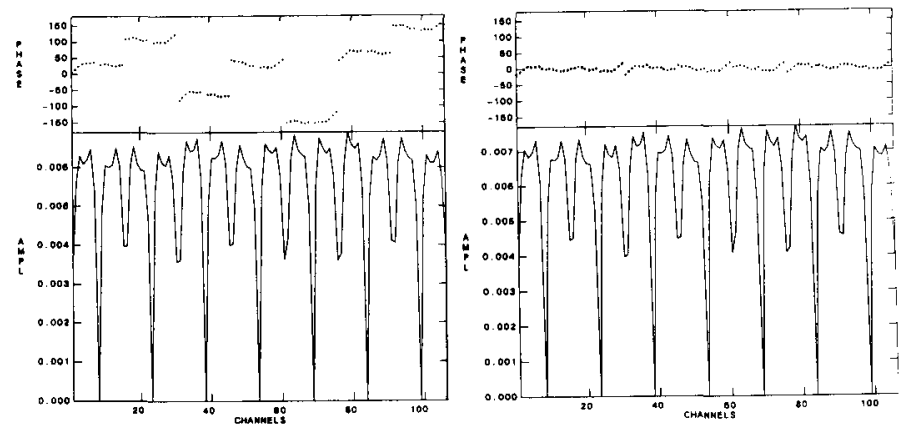

Fig. 1. Amplitude and phases across the bandpass for the baseline Effelsberg-Westerbork when observing OQ208. Left panel shows the original data, right panel shows the data after fringe fit.

sensitivity. The experiment described in this paper was made at $18 \mathrm{~cm}$ with the EVN.

The Mk3 system has a wider bandwidth and therefore higher sensitivity than the previously used Mk2 system, but it also has a smaller field of view. Thus we intended to use bandwidth synthesis technique to increase the field of view while retaining the sensitivity. The original plan was to fit for fringes in the standard way for Mk3 data, and then grid the IF-channels separately to get a wider field of view.

At that time the effort to find more fringes from $3 \mathrm{~mm}$ VLBI had started and the MK3IN-program and global fringe fitting of Mk3 data (Bàath these proceedings) were under development. Therefore we decided to wait with the data reduction until this data reduction technique could be used on the $1422+202$ data as well.

The reduction could be started in 1989 and we soon found that there was a problem with the phase-cal signal at one of the stations and therefore the technique described in Bååth (these proceedings) could not be directly used for finding fringes. Fortunately we had also observed the source OQ208 as an amplitude calibrator every 3 hours. We decided to fit for fringes for every IFchannel independently on the calibrator and then apply the solution on the target source $1422+202$. This technique would be equivalent to using a phasecal signal, and would allow us to thereafter fit for multi-and single band delays on $1422+202$.

However, we had the solution on OQ208 applied to $1422+202$ at this stage, and decided to map the data on $1422+202$ without any further fringe solution. The source showed up, but with a position offset which later showed to be correct. The image was, to our surprise, quite well focused, with a well defined compact component coinciding with where the core ought to be. We then proceeded to fit for station based phase offsets in order to further focus the image. This selfcalibration step did not change the position of the compact core, but served to make a more focused image. Later we also made an image using the standard baseline fitting technique. The two images are shown together in Fig. 2. The image made by the standard technique is smeared due 

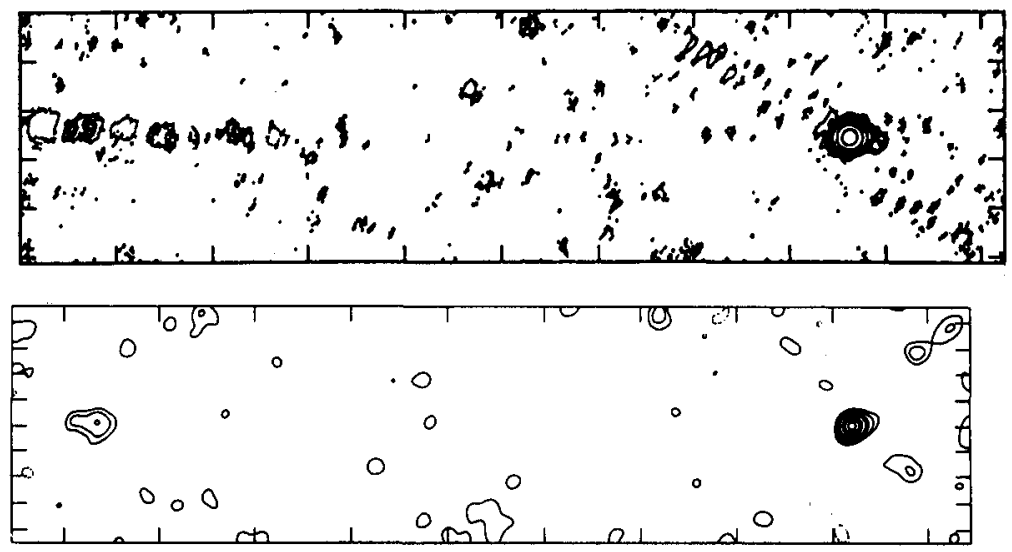

Fig. 2. Maps of $1422+202$ made with the standard baseline fring fitting technique (top), and our new technique (bottom). The restoring beam was $0 . " 2$ in both cases.

to the wide bandwidth, while the image made using our new technique does not suffer from this effect. Both images are shown convolved with a larger beam than natural. This is to more easily identify components which are smeared as described above.

Our map can now be compared to a VLA map, and the positions can be directly identified since both images uses OQ208 as reference position on the sky. Fig. 3 shows sections of our map together with a VLA map. The cross indicate the position and error bars of the optical identification. It is clearly seen that the core in our map is situated at the edge of the VLA core rather than in its center, and in very close agreement with the optical image. We have also detected the southern lobe, but it is mostly resolved on our longest baselines.

We want to stress that we did not design our experiment as a phase referencing experiment. It just turned out that way. The distance between the two sources is $9 .^{\circ} 6$, much larger than has been believed duable. Our experiment was done at night time and with the EVN only, but the fact that this probably could not be done at day should not stop us from doing it at night!

Our experiment shows that it is possible to phase reference sources at distances larger than $9^{\circ}$ from each other with the EVN. We might have had some luck, in that the ionosphere might have been particularly cooperative, but even including that we now believe that the EVN may be a much more stable instrument than has been believed up till now. We suggest that the EVN, at night time and at 18 and $6 \mathrm{~cm}$, can and should be extensively used for phase reference projects. A properly designed phase reference experiment should also switch betwen the reference and target sources on much short time scales. A faster switching would result in a better focused image than in our case. 


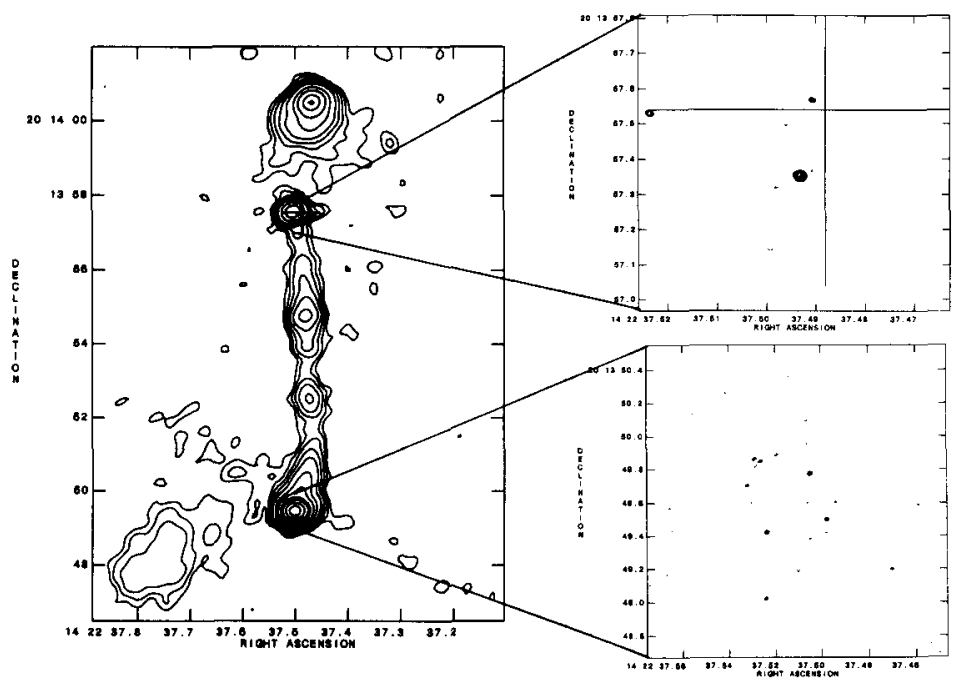

Fig. 3. A VLA map of $1422+202$ (left) and the core part (upper right) and southern lobe part (lower right) from our VLBI map. Crosses indicate the position of the optical identification. 